\title{
A NOVEL TECHNIQUE OF MINIMALLY INVASIVE RETRO- RECTUS REPAIR OF VENTRAL HERNIA- MODIFIED ETEP
}

Poster presented at Forum for Interdisciplinary Research in Medicine, Science \& Technology Conference, Moscow 2020

Peer reviewed by JS Bamrah, Ananthakrishnan Raghuraman, Soumit Dasgupta \& Jyothi Srinivasan

Keywords: hernia repair; retro-rectus repair

Aim:

To compare the clinical effectiveness of eTEP technique versus conventional IPOM repair for ventral hernia. We also aim to introduce our innovative Kumar-Subhash's modified 3 port eTEP technique for repair of ventral hernias.

\section{Introduction:}

Ventral hernia repair is one of the common general surgical procedures. A novel technique has been developed where in a mesh is placed in retro muscular plane by minimal access technique - Extended totally extraperitoneal repair (eTEP). There is scarcity of data on its effectiveness when compared to conventional laparoscopic intraperitoneal onlay mesh (IPOM) hernioplasty.

\section{Materials and Methods:}

A prospective comparative study was undertaken at Devagiri Hospital, Bangalore, India from January 2017 to December 2019. 30 patients were included in the study based on predefined inclusion and exclusion criteria. Patients were divided into two groups, the eTEP group comprised of 12 patients and 18 patients were included in IPOM group. Since, we adopted a new technique, patients' choice of procedure was respected and hence, no randomisation was done. Appropriate statistical analysis of the collected data was done. The outcomes were compared with respect to postoperative pain, duration of hospital stay and postoperative complications.
Anil KUMAR 1, Meghana TAGGARSI 2, Subhash RC 3

1 Stepping Hill Hospital, Stockport NHS

Foundation Trust, Stockport, United Kingdom 2 Royal Blackburn Hospital, Blackburn, United Kingdom

3 Devagiri Hospital, Bangalore, India

correspondence to nettyanil@gmail.com

Article Information

DOI: 10.38192/1.6.3.firmst20.ab36

Epub: 20.09.2020

Presented at FIRMST Conference, Moscow 2020

Peer reviewed by JS Bamrah, Ananthakrishnan Raghuraman, Soumit Dasgupta

Open Access- Creative Commons Licence CCBY-ND-4.0

\section{Results:}

Both groups were comparable with respect to age, gender and presenting complaints. The mean pain score on postoperative day (POD) 1 in eTEP group was 3 , and 1 on POD 7 while the IPOM group had a mean pain score of 7 and 3 on POD 1 and POD 7 respectively which was statistically significant. Patients in eTEP group had a shorter length of hospital stay. None of the patients in eTEP group had any complications related to the novel technique.

\section{Conclusion:}

Our study demonstrates that Kumar-Subhash's modified 3 port eTEP technique is a novel and easy approach for laparoscopic repair of ventral hernia especially umbilical hernia and offers an additional skill to the armamentarium of Minimally Invasive surgeons. It is a feasible, effective and safe option for repair of ventral hernias with good clinical and cosmetic outcomes for patients.

Conflict of Interests/Comments: Nothing to declare. 